\title{
BMJ Open Public health surveillance of automated external defibrillators in the USA: protocol for the dynamic automated external defibrillator registry study
}

\author{
JoAnn Broeckel Elrod, ${ }^{1}$ Raina Merchant, ${ }^{2,3}$ Mohamud Daya, ${ }^{4}$ Scott Youngquist, ${ }^{5}$ \\ David Salcido, ${ }^{6,7}$ Terence Valenzuela, ${ }^{8,9}$ Graham Nichol ${ }^{1}$
}

To cite: Elrod JAB

Merchant R, Daya M, et al. Public health surveillance of automated external defibrillators in the USA: protocol for the dynamic automated external defibrillator registry study. BMJ Open 2017;7:e014902. doi:10.1136/bmjopen-2016014902

- Prepublication history for this paper is available online. To view these files please visit the journal online (http://dx.doi.org/10.1136/ bmjopen-2016-014902).

Received 25 October 2016 Revised 10 February 2017 Accepted 27 February 2017

CrossMark

For numbered affiliations see end of article.

Correspondence to Professor Graham Nichol; nichol@uw.edu

\section{ABSTRACT \\ Introduction: Lay use of automated external defibrillators (AEDs) before the arrival of emergency medical services (EMS) providers on scene increases survival after out-of-hospital cardiac arrest (OHCA). AEDs have been placed in public locations may be not ready for use when needed. We describe a protocol for AED surveillance that tracks these devices through time and space to improve public health, and survival as well as facilitate research.}

Methods and analysis: Included AEDs are installed in public locations for use by laypersons to treat patients with OHCA before the arrival of EMS providers on scene. Included cases of OHCA are patients evaluated by organised EMS personnel and treated for OHCA. Enrolment of $10000 \mathrm{AEDs}$ annually will yield precision of $0.4 \%$ in the estimate of readiness for use. Enrolment of 2500 patients annually will yield precision of $1.9 \%$ in the estimate of survival to hospital discharge. Recruitment began on 21 Mar 2014 and is ongoing. AEDs are found by using multiple methods. Each AED is then tagged with a label which is a unique two-dimensional (2D) matrix code; the 2D matrix code is recorded and the location and status of the AED tracked using a smartphone; these elements are automatically passed via the internet to a secure and confidential database in real time. Whenever the $2 \mathrm{D}$ matrix code is rescanned for any non-clinical or clinical use of an AED, the user is queried to answer a finite set of questions about the device status. The primary outcome of any clinical use of an AED is survival to hospital discharge. Results are summarised descriptively.

Ethics and dissemination: These activities are conducted under a grant of authority for public health surveillance from the Food and Drug Administration. Results are provided periodically to participating sites and sponsors to improve public health and quality of care.

\section{INTRODUCTION}

Out-of-hospital cardiac arrest (OHCA) is defined as a sudden and unexpected pulseless
Strengths and limitations of this study

- Study tracks individual automated external defibrillators over time.

- Outcome is ascertained by public health surveillance.

- Study has potential for selection bias due to incomplete case finding.

condition attributable to cessation of cardiac mechanical activity. ${ }^{1}$ Cardiac arrest has multiple aetiologies, and the aetiology of arrest influences treatment decisions. Underlying mechanisms for non-traumatic cardiac arrest are crudely categorised as (1) conductive abnormalities of the myocardium leading to arrhythmias, (2) chronically weakened myocardium leading to end-stage pump failure and (3) acute occlusion of a coronary artery leading to myocardial infarction. Of these mechanisms of OHCA, resuscitation is generally most successful for isolated conductive abnormalities or for acute coronary thrombosis that is treated rapidly. Acute occlusion is common among patients with a first recorded rhythm of ventricular fibrillation (VF), which hereafter includes pulseless ventricular tachycardia as well as rhythms interpreted as shockable by an automated external defibrillator (AED). Recognition ${ }^{2}$ and successful treatment of VF is highly time-dependent. ${ }^{3}$ AEDs are medical devices (MDs) intended to simplify and improve treatment of OHCA. The timely use of AEDs by laypersons in conjunction with cardiopulmonary resuscitation (CPR including manual chest compressions with or without ventilation) is the only field intervention that has been shown to significantly increase the number of individuals who survive to discharge after OHCA. ${ }^{4}$

Since the effectiveness of use of AEDs by laypersons was demonstrated more than a 
decade ago, more than 2.4 million AEDs have been sold for use by laypersons in the USA. ${ }^{5}$ As more of these AEDs are placed in the community, the Food and Drug Administration (FDA) has received reports of potential adverse events associated with them. Since the overall number of AEDs installed is not public information and it is difficult to track individual AEDs through time and space, agency staff have experienced challenges in trying to interpret these reports. ${ }^{6}$ As well, each major manufacturer of AEDs in the USA has recalled their devices to address one or more potential safety issues over the past 5 years. A need for a more comprehensive surveillance system has been identified. ${ }^{7}$ Specifically, FDA's advisory panel recommended "Registry information should be established to collect more data. An active reporting registry would help with recalls and would help to maintain the quality and confidence of the data collected. Sold devices could be tracked prospectively, and long term device performance would be captured."

In this paper, we describe the rationale for and design of an ongoing dynamic registry for surveillance of AED location and use. This registry has been created through a partnership of academics, FDA, key stakeholders and manufacturers of AEDs. The long-term goals of the registry are to provide reliable, valid and sustainable postmarket surveillance of AEDs; to provide important and timely information to patients, providers and the public to improve MD device development including the quality of AEDs and the quality of care for patients with OHCA and to provide a framework for prospective embedded studies of the effectiveness of next-generation AEDs. The Dynamic AED Registry represents a novel approach to AED surveillance, leverages a clinical registry infrastructure and can be applied to other MDs that are mobile in time and space.

\section{Methods and analysis}

Study populations

Included AEDs are those:

A. Pre-existing in community settings and

I. Identified by crowdsourcing techniques, or

II. Identified by manufacturer or distributor.

B. Newly installed in community setting:

I. Identified by owner during at time of installation, or

II. Identified by manufacturer at time of sale.

Included patients are those treated for OHCA:

A. Evaluated by organised emergency medical services (EMS) personnel and receive attempts at external defibrillation (by lay, police or EMS), or

B. Receive chest compressions by organised EMS personnel.

Although a minority of patients with OHCA have an AED applied by a layperson before the arrival of EMS providers on scene (ref 8, p. 339), we monitor all cases of EMS-treated cardiac arrest in participating communities to obtain complete case finding. Monitoring the outcome of every AED use is necessary to identify those uses that may have caused or contributed to survival or death (21CFR803), since the majority of patients treated for OHCA die.

To date, studies reported from smaller selective registries were difficult to interpret because of potential selection bias and lack of knowledge of the overall population. This potential selection bias should be mitigated as the Dynamic AED Registry evolves over time to include a high percentage of treated patients in the USA.

Several strategies are used to achieve a high rate of enrolment in the Dynamic AED Registry. Initially, AEDs were enrolled by their identification in community settings by using crowdsourcing techniques. ${ }^{9}$ According to these methods, individuals or teams of volunteers register to participate in a scavenger hunt contest. Those who identify the most AEDs during the contest period receive a prize. As well, the first individual or team to report one of a finite number of preselected AED receive a prize.

Now, enrolment has been extended to include prospective registry of AEDs sold by a manufacturer for use by lay persons in public locations. We also collate information about individual AEDs reported by laypersons who have registered online and then used their smartphone (https://heartmap.uwctc.org/, accessed on 25 May 2016). Individual device identifiers are provided by the investigators to owners or responsible individuals of these AEDs, with information about the purpose and methods of AED surveillance, as well as instructions on where to apply the identifier to the AED and how to register the AED in the dynamic registry by scanning the identifier. A series of individual identifiers are provided to each sponsoring manufacturer for inclusion with the product registration materials provided with AEDs newly sold for use in community settings.

Organisations that provide physician oversight and AED readiness services encourage client participation by placing an individual identifier on each AED under their supervision then scanning the identifier to record information about the location of the device in our secure database. With permission of the client, this information is shared with the relevant EMS dispatch centre so as to improve the community response to OHCA for the client's staff and customers. The lightweight and portability of AEDs makes them easily movable. If the AED is moved later and then the identifier is rescanned, we provide information about the updated location of the device to the oversight organisation so that the location of the AED is known at all times.

Participating EMS agencies are recruited as interest and resources allow. Initially, this included a mix of EMS agencies that participated in the Resuscitation Outcomes Consortium (funded by the National Institutes of Health, American Heart Association and other agencies), are participating in the Mission:Lifeline Cardiac Resuscitation program (funded by American Heart Association) or associated with a dispatch centre using 
software to enable faster lifesaving AED response within the community (PulsePoint Responder, PulsePoint Foundation, San Ramon, CA). These vanguard sites included Philadelphia, PA, Pittsburgh, PA, Portland, OR, Salt Lake City, UT, Seattle, WA and Tucson, AZ. We expect that participation will expand over time to include as many EMS agencies as possible throughout the USA. Furthermore, EMS agencies or receiving hospitals may enrol patients to provide an analysis of their programme as well as to benchmark the process and outcome of care compared to other participant's experience.

\section{Data acquisition}

EMS providers and personnel responsible for maintenance of AEDs are encouraged to scan the individual identifier after each AED use. On scanning of the 2D matrix code, the date, time and location of use are automatically identified, recorded and transmitted to the dynamic registry. As well, users are asked to respond to a finite set of queries confirming the location of the AED as well as describing the reason for its scan. In the event of a clinical use, EMS providers who scan the individual identifier are queried about the patient's intended hospital destination to facilitate subsequent tracking of patient vital status at hospital discharge. After the event, EMS providers are queried about a finite set of patient and EMS factors to facilitate adjustment of outcomes for case-mix as well as assessment of rates of potential adverse events in a manner consistent with the Utstein template for reporting of outcomes after OHCA. ${ }^{1}$ As well, investigators ascertain the participant's vital status at discharge from the receiving hospital. Anonymised quarterly reports are provided to participating EMS systems, which describe the process and outcome of care for patients with OHCA as an incentive to facilitate continued surveillance of AEDs, their use and factors that contribute to positive clinical outcomes. The quality of the data included in the registry is monitored and improved by using regular checks and audits.

\section{Data elements}

Baseline characteristics, covariates and outcomes included in the registry were developed and implemented by consensus of the executive committee. In doing so, emphasis was placed on developing a finite set of key variables so as to ensure that the registry was simple, sustainable and scalable. These can be revised periodically as required.

\section{Data quality control}

The Dynamic AED Registry uses multiple mechanisms to ensure data completeness and accuracy (table 1). Monitoring and auditing use standardised data quality checks. Events are adjudicated as required for prespecified outcomes.

\section{Use of data to improve care}

The Dynamic AED Registry provides feedback to participating sites including periodic quality benchmarking. Participants have access to a repository of their own data and tools to evaluate their local practice and conduct user-specified local data queries. This is especially valuable for communities that alert lay responders to the need for bystander CPR and AED need through dispatch-identified OHCA. Data are also provided to FDA to address specific surveillance questions.

\section{Use of data to facilitate research}

The data will be used to facilitate monitoring of enrolment in prospective embedded evaluations of interventions in patients with cardiac arrest. For example, the majority of vanguard sites are planning to participate in large randomised trials of resuscitation interventions as soon as feasible in patients treated for OHCA. As sites implement this trial, trial investigators can monitor whether all eligible patients were enrolled by comparing the volume of patients enrolled in the AED registry to that enrolled in the trial. As well, the characteristics of the patients who accrue to the trial can be compared to data obtained from the AED registry. In order to adhere to patient privacy requirements, data from the registry will not be linked to individual patients who might also be enrolled in the clinical trial. Instead, aggregate statistics for the population of all patients with OHCA treated within the study communities will be compared to aggregate statistics for the patients enrolled in this trial. From

Table 1 Techniques for optimising data quality control

\begin{tabular}{lll}
\hline & $\begin{array}{l}\text { Applied to all dynamic AED } \\
\text { Registry Data }\end{array}$ & $\begin{array}{l}\text { Applied to } \\
\text { postapproval studies }\end{array}$ \\
\hline $\begin{array}{l}\text { Site training and support from Registry staff for queries } \\
\begin{array}{l}\text { Data cleaning: data integrity checks using range validation } \\
\text { and other measures }\end{array}\end{array}$ & $\begin{array}{l}\text { Yes } \\
\text { Audit portion of data }\end{array}$ & Yes \\
$\begin{array}{l}\text { Collection of source documents and verification of } \\
\text { prespecified key events }\end{array}$ & Yes & Yes \\
$\begin{array}{l}\text { Adjudication of selected outcomes } \\
\text { Potential adverse events }\end{array}$ & No & Yes \\
& $\begin{array}{l}\text { Yes } \\
\text { No shock advised by AED but } \\
\text { first EMS rhythm shockable }\end{array}$ & Yes \\
\hline
\end{tabular}


such a comparison, the generalisability of the trial results can be investigated.

\section{INNOVATIVE METHODS}

The Dynamic AED Registry uses multiple innovative methods to enhance surveillance of AEDs.

\section{Crowdsourcing}

We have adapted crowdsourcing methods to find AEDs installed in public locations for use by laypersons in major metropolitan areas that are participating in the Dynamic AED Registry. Merchant pioneered use of crowdsourcing to identify AEDs installed in community settings. ${ }^{9}$ According to these methods, help is solicited for tasks usually performed by particular individuals from an undefined large group (ie, a crowd). Since the task is offered to a large group with diverse backgrounds, it has the potential to attract individuals who are interested in the problem, likely to finish the task, and likely to contribute innovative ideas. On the basis of methods developed by the Defense Advanced Research Projects Agency (DARPA), ${ }^{10}$ adults were invited to identify AEDs installed in community settings in Philadelphia County, Pennsylvania. According to state law, AEDs installed in community settings in Philadelphia County, Pennsylvania, should be registered with the local EMS authority. Before the contest began, 57 devices were registered with Philadelphia EMS. Monetary prizes were awarded to those individuals or teams who reported the most AEDs, as well as to those who were first to report any of 200 preselected AEDs. During the contest, 313 individuals and teams identified 852 unique AEDs in 528 locations. These locations were in $94 \%$ of eligible census tracts and included a mix of public (59\%) and private $(41 \%)$ locations. $50 \%$ of AEDs were identified as either non-functional or having unknown functional status. The investigators concluded that crowdsourcing is a feasible approach for identifying AEDs installed in community settings in a large urban city.

\section{Able to track individual medical devices}

We have pioneered the use of an individual identifier to track AEDs or other MDs through time and space in community settings. According to this method, each AED is tagged with a label which consists of a unique two-dimensional (2D) matrix code ( $\mathrm{QR}$ code, Denso Wave, Chita-gun, Japan); then the QR code or 2D matrix code is recorded as well as the location and status of the AED by using open-source software native to any contemporary smartphone (eg, Google Goggles, Google, Mountain View, CA) and finally these elements are automatically passed via the internet to a secure and confidential database in real time (Provisional Patent \# $61 / 498424$, figure 1). The user is prompted to rescan the label after any use (figure 2). Note that by design each label has a unique alphanumeric code that provides built in redundancy with the $2 \mathrm{D}$ matrix code. The user is queried to answer a finite set of questions when the smartphone is connected to the database (table 2). If the device has a clinical use, these queries include verification of the intended receiving hospital for transported cases to facilitate longitudinal follow-up of patient outcome to hospital discharge (table 3). Using the limited data collected in the field with the 2D matrix code, additional variables are abstracted later from the participant's record to further characterise the process and outcome of care.

The method for scanning the 2D matrix code is deliberately platform independent (ie, agnostic). We opted to design and implement a website that can be accessed by any web-capable smartphone rather than take on the burden of designing, disseminated and maintaining multiple different smartphone applications. Most EMS providers carry their own smartphone. Since no specific smartphone application is required to access the website, our web developer is able to update this centrally as needed.

This device identifier complement's the FDA's Global Use Device Identification Database (GUDID) initiative. ${ }^{11}$ This establishes that a unique device identifier number is assigned by the device manufacturer to each version or model of a device. This identifier is in humanreadable format and in machine-readable format. It is intended to contain information about the type of device as well as information about its manufacture. The primary difference is that GUDID does not require or enable tracking of the location of a device through time and space.

\section{Sample size and analysis}

Recruitment began on 21 Mar 2014. Enrolment of AEDs and patients with OHCA in this cohort study is ongoing. There is no planned end. As such, there is no maximum expected enrolment necessary to detect a significant difference between groups, as there would be in a clinical trial. The primary analysis will be descriptive. If $95 \%$ of AEDs are ready for use, enrolment of 10000 AEDs

Figure 1 Use of unique device identifier for AED surveillance. AED, automated external defibrillator.

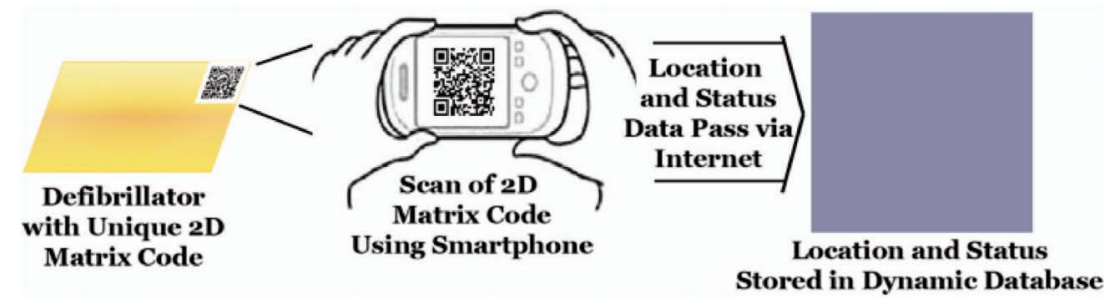




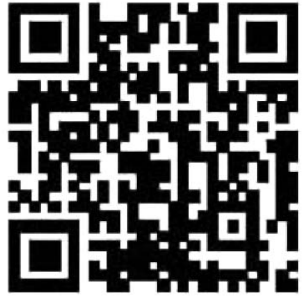

Help Save

Lives!

Scan QR code

AFTER use

or service.

aed.uwctc.org $/ \mathrm{m} / 8 \mathrm{fbg} 5 \mathrm{cb}$

Figure 2 Two-dimensional matrix code for AED tagging. AED, automated external defibrillator.

Table 2 Questions for EMS on scanning after clinical use

\begin{tabular}{|c|c|}
\hline Question & Definition \\
\hline Device not used & $\begin{array}{l}\text { Medic, bystander or other person tried } \\
\text { to apply AED to patient but was unable } \\
\text { to use device; reason device not used }\end{array}$ \\
\hline $\begin{array}{l}\text { Date of cardiac } \\
\text { arrest }\end{array}$ & Date cardiac arrest occurred \\
\hline $\begin{array}{l}\text { Time of cardiac } \\
\text { arrest }\end{array}$ & Time cardiac arrest occurred \\
\hline Age & Patient's age in years \\
\hline Gender & Patient's gender \\
\hline $\begin{array}{l}\text { Destination } \\
\text { hospital }\end{array}$ & $\begin{array}{l}\text { Hospital to which patient was } \\
\text { transported }\end{array}$ \\
\hline $\begin{array}{l}\text { First responder } \\
\text { agency }\end{array}$ & $\begin{array}{l}\text { Name and state-assigned code } \\
\text { number from first responder vehicle }\end{array}$ \\
\hline
\end{tabular}

Table 3 Patient outcome variables

\begin{tabular}{ll}
\hline Variable & Definition \\
\hline $\begin{array}{l}\text { Emergency } \\
\text { department } \\
\text { disposition }\end{array}$ & $\begin{array}{l}\text { Final disposition of patient from } \\
\text { emergency department: patient } \\
\text { documented to be discharged from ED } \\
\text { alive, patient documented to die in ED, } \\
\text { transferred to a different hospital, }\end{array}$ \\
$\begin{array}{l}\text { admitted to same hospital. } \\
\text { Vital status at }\end{array}$ & $\begin{array}{l}\text { Patient's vital status at discharge from } \\
\text { discharge }\end{array}$ \\
$\begin{array}{l}\text { hospital: died in hospital, discharged } \\
\text { alive, not yet determined. }\end{array}$ \\
at discharge & $\begin{array}{l}\text { Patient's neurologic status based on } \\
\text { CPC at discharge from hospital: good } \\
\text { cerebral performance, moderate }\end{array}$ \\
& $\begin{array}{l}\text { cerebral disability, severe cerebral } \\
\text { disability, coma/vegetative state. }\end{array}$ \\
\hline CPC, cerebral performance category.
\end{tabular}

annually will yield precision (measured as half of the $95 \% \mathrm{CI})$ of $0.4 \%$ in the estimate of readiness for use. If $10 \%$ of patients treated for OHCA survive to discharge, enrolment of 2500 patients annually will yield precision of $1.9 \%$ in the estimate of survival to discharge.

\section{Dissemination}

The Dynamic AED Registry is the first registry to be conducted under a grant of authority for public health surveillance from FDA. As such, these activities have been determined by multiple institutional review boards (IRBs) to not be subject to human subjects' regulations and to exercise the public health exception within the Health Insurance Portability and Accountability Act (HIPAA, 45 CFR 164.512(b)). These methods are adaptable to public health surveillance of other MDs.

\section{DISCUSSION}

Lay use of AEDs before the arrival of EMS providers on scene increases survival after OHCA. ${ }^{4}$ Large numbers of AEDs have been placed in public locations to improve the community response to cardiac arrest. ${ }^{5}$ It is sometimes difficult to determine whether these devices are maintained in a state of readiness for use or whether they functioned as intended when applied to someone in presumed cardiac arrest. Importantly, there is no widely deployed method of tracking their location and use in community settings. We describe the implementation and maintenance of a dynamic registry for surveillance of AEDs through time and space.

This registry serves multiple purposes, including public health surveillance of AEDs, quality improvement of AED use as well as overall EMS care, and serving as a framework for embedded research studies. We note that local and multicentre registries of patients with OHCA are often used for multiple purposes. For example, the ROC cardiac arrest registry ${ }^{12}$ measured the public health impact of cardiac arrest in participating communities ${ }^{13}$ provided a mechanism for local quality improvement $^{14}$ as well as a mechanism for embedded randomised trials. ${ }^{15-17}$ Unfortunately funding for ROC has ceased, and the ROC registry collated too many variables to be sustainable over the long term without dedicated funding.

The Dynamic AED Registry has several strengths. By design, it is simple, sustainable and scalable. Since the registry functions under a grant of authority for public health surveillance, the base surveillance and quality improvement functionality is not subject to human subjects' research regulations and is exempted from HIPAA. Data collected for public health surveillance can, subject to appropriate permissions, can be used to improve the process and outcome of care for patients with OHCA in participating communities. Like other registries, it can also be used as a framework for embedded prospective research evaluations of existing or next-generation defibrillators, as well as other interventions intended to improve outcomes after OHCA.

What does the Dynamic AED Registry offer over other registries? It tracks the location and use of individual AEDs in diverse communities. This identifies the specific AED used, allowing verification of the diagnostic algorithm in no-shock advised cases. It supports passive 
reporting of potential AED failure that is supposed to occur. AED manufacturers have recognised the value of and subsequently invested in the Dynamic AED Registry to provide ongoing safety and effectiveness data, which can be useful as real-world evidence to support regulatory decisions for AEDs. Recognising that data collated in different resuscitation registries may not be comparable, the academic leadership of the Dynamic AED Registry is working with leaders of other resuscitationrelated registries to encourage harmonisation of data elements, quality control techniques, outcomes and adverse event classification.

This registry has some limitations. The robustness of the data collated by it depends in part on the completeness of case finding by EMS providers and participating sites may have variable success in enrolling consecutive AEDs or patients. On the basis of a comparison of patients not enrolled versus enrolled in a registry intended to enrol consecutive patients with acute coronary syndrome, patients who are not included in a registry may have higher risk, receive poorer quality of care and have a higher mortality than those who are included. ${ }^{18}$ Such selection bias limits the ability of registries to reliably assess the effectiveness of study interventions. ${ }^{19}$

At present, the Dynamic AED registry has a small footprint but this is expected to grow over time. Although crowdsourcing methods are used to identify AEDs that were placed in public locations, it is not feasible to apply crowdsourcing in every urban and rural community. Instead, the registry is evolving to include prospective tagging of AEDs through manufacturers as they are placed in public locations. The distribution of AEDs by geography may be non-random (ie, one manufacturer may have more devices placed in a given community than another). Since geographic region is a strong predictor of outcome after OHCA, ${ }^{20}$ comparison of outcomes by different manufacturers needs to account for differences in geography.

The small footprint of the registry impacts on its representativeness and may be associated with selection bias. Communities choose to participate or not participate in the registry. To the extent that participating communities have greater (or lesser) survival than other communities, the registry may over (under) estimate the effectiveness of AEDs.

It is plausible that incomplete identification and scanning of QR codes may impact on the registry's representativeness and may be associated with selection bias. To try to mitigate this, participating communities are encouraged to track and report all patients with OHCA treated by EMS providers, regardless of whether they are initially identified as having been treated with an AED by a layperson.

The Center for Devices and Radiological Health (CDRH) of the US FDA of the USA is responsible for regulating firms who manufacture, distribute or import MDs sold in the USA. MDs are instruments or related articles which are intended for use in the diagnosis or treatment of disease, are applied externally or internally to patients to affect the structure or function of their body and do not achieve their purpose through chemical action or metabolism. ${ }^{21}$ FDA relies on registries for postmarket surveillance of MDs to ensure their safety and efficacy. The US congress has directed the agency to implement an active system capable of identifying safety issues and communicating them in a timely manner to patients, providers and the public. ${ }^{22}$ Policymakers have suggested that increased surveillance of MDs is necessary to enhance and maintain patient safety. ${ }^{23}$ The Institute of Medicine (IOM) made recommendations about how to improve the approval process and postmarket surveillance of MDs. ${ }^{24}$ As well, the IOM made recommendations about how to improve outcomes after OHCA that included the establishment and maintenance of a sustainable method of national surveillance of the process and outcome of care of all patients with OHCA. ${ }^{25}$ Experts have recommended postmarket surveillance of any AED introduced for in-hospital, emergency medical services or public access defibrillation. ${ }^{26}$

Our registry can be placed in the context of FDA's evolving medical device regulatory process (table 4) as well as in the context of other resuscitation-related registries. It is a next-generation registry implemented with the multiple purposes of surveillance of MDs as well as measurement and improvement of the process and outcome of care for patients in real-world settings, as well as serving as a framework for embedded studies of the effectiveness of defibrillator as well as other interventions in patients with OHCA. The Dynamic AED Registry is designed to evolve into a comprehensive but scalable and sustainable national registry in the USA. Multiple other resuscitation-related registries exist in the USA, and the rest of the world that seek to characterise the process and outcome of care related to OHCA. ${ }^{27}$ However, to the best of our knowledge, none are capable of tracking clinical and non-clinical use of individual AEDs including vital status at hospital discharge. As well, some have experienced challenges related to sustainability.

Multiple other registries exist that describe the process and outcome of care for patients with OHCA. In the USA, the Resuscitation Outcomes Consortium (ROC) Cardiac Arrest Registry enrolled such patients in geographic sites that were selected by a competitive process to participate in a clinical research network. ${ }^{12}$ This registry included all patients with non-traumatic OHCA assessed or treated by participating EMS providers. Data reported by participating EMS agencies were routinely screened for missing cases by comparing the observed number of cases in any month to the expected number for that agency as estimated by averaging the observed cases over a 12-month period. As a consequence of this, the incidence of EMS-treated OHCA enrolled in this registry increased by $25 \%$ over time. ${ }^{13} 28$ The registry used multiple methods to monitor and improve data quality and completeness. It was intended to facilitate 
Table 4 Dynamic AED Registry in context of FDA approval process for medical devices

\begin{tabular}{|c|c|c|c|c|}
\hline & \multirow[b]{2}{*}{ Premarket } & \multirow{2}{*}{\multicolumn{3}{|c|}{$\begin{array}{l}\text { Hybrid } \\
\text { Dynamic AED Registry }\end{array}$}} \\
\hline & & & & \\
\hline & Phase II & Phase III & Postapproval & Postmarket Clinical registry \\
\hline Aim & Efficacy & \multicolumn{3}{|c|}{ Safety, efficacy and effectiveness } \\
\hline Inclusion criteria & Restrictive & Either & \multicolumn{2}{|l|}{ Broad } \\
\hline Intervention & Tight protocol & Either & \multicolumn{2}{|c|}{ Implemented in usual clinical practice } \\
\hline Cointervention & $\begin{array}{l}\text { Based on protocols for } \\
\text { many aspects of care }\end{array}$ & Either & \multicolumn{2}{|c|}{$\begin{array}{l}\text { Based on local practice; monitored but } \\
\text { minimal control }\end{array}$} \\
\hline Adherence to protocol & Required & Either & \multicolumn{2}{|c|}{$\begin{array}{l}\text { Expected and considered in sample size and } \\
\text { analysis plan }\end{array}$} \\
\hline Events & $\begin{array}{l}\text { Related to biological } \\
\text { effect (eg, conversion out } \\
\text { of shockable rhythm) }\end{array}$ & Either & & $\begin{array}{l}\text { Related to patient outcome } \\
\text { (eg, survival) }\end{array}$ \\
\hline Analysis & Treatment received & Both & \multicolumn{2}{|c|}{ Intention to treat } \\
\hline Sample size & Usually $<1000$ & Either & \multicolumn{2}{|c|}{ Usually $>1000$} \\
\hline Data burden & Large & $\begin{array}{l}\text { Core supplemented by } \\
\text { study-specific }\end{array}$ & \multicolumn{2}{|c|}{ Minimal and simple core } \\
\hline Study management & $\begin{array}{l}\text { Significant interventions } \\
\text { and support from } \\
\text { research staff }\end{array}$ & \multicolumn{3}{|c|}{ Minimal support and interventions from research team } \\
\hline
\end{tabular}

case identification and data collection for ROC intervention trials so it used more than 200 variables to characterise each enrolled episode. The registry informed the design and implementation of multiple pragmatic trials in patients with OHCA, ${ }^{15} 1729-31$ and secondary analyses about the association between patient and treatment characteristics. ${ }^{13}$ 32-40 Although funding to support data collection in US ROC sites has ceased, most sites are continuing to enrol cases in this registry during ROC's current no-cost extension phase. We adapted and extended the ROC registry as a simple, sustainable, registry focused on tracking the process and outcome of care associated with use of AEDs.

The Cardiac Arrest Registry to Enhance Survival (CARES) Registry initially focused on enrolment of cardiac arrest of presumed cardiac aetiology, but has broadened its inclusion criteria to include cardiac arrest of any aetiology. ${ }^{41}$ Since there is at least a twofold variation in the reported proportion of cardiac arrests that are of non-cardiac aetiology, ${ }^{42}$ assessments of the effectiveness of interventions for OHCA that include only cases with presumed cardiac aetiology may be susceptible to selection bias. Reports from CARES emphasise survival among patients with witnessed VF rather than among all treated patients. Importantly, the likelihood that an initial rhythm will be VF depends in part on the time of rhythm assessment. ${ }^{2}$ A consequence of emphasising survival in this subpopulation rather than all patients treated for OHCA is that assessments of the effect of interventions intended to improve survival (eg, AEDs) may be susceptible to bias or confounding. CARES reports that 32 states contribute data to it, but the catchment population within each ranges from $50 \%$ to $100 \% .{ }^{44}$ As well, multiple EMS agencies that participate in CARES report that $100 \%$ of their cases received bystander CPR and $100 \%$ survived to discharge, which suggests that they may be missing cases that did not receive bystander CPR (and did not have a good outcome) (ref. 44, p. 16 and 17). Collectively, these observations suggest that the external validity of CARES data may require further assessment. CARES was initially funded by the Centers for Disease Control. It is currently funded by the American Heart Association and Medtronic Foundation, as well as a subscription-based funding model.

The National Emergency Medical Services Information System (NEMSIS) is an electronic documentation system that is intended for use in every local EMS system. ${ }^{45}$ Local data are collated by each state or territory and then contributed to a national data warehouse. Over $90 \%$ of states and territories have a NEMSIS-compliant system. ${ }^{46}$ Challenges to use of NEMSIS for public health surveillance of AEDs include that many states are working to revise data elements, improve data capture and ensure compliance with the latest NEMSIS data standard. Another challenge is that historically NEMSIS has had limited hospital outcome data. NEMSIS is supported by state public health departments.

The PulsePoint program incorporates information about AED locations (as opposed to individual AEDs) into dispatch software used by Public Safety Answering Points to improve the community response to OHCA. The Dynamic AED Registry provides location information to PulsePoint to supplement and periodically update their location information. When a dispatcher identifies that someone has had OHCA in a community that is participating in the PulsePoint program, citizens who have previously downloaded a location-aware application to their smartphone are alerted to the event if they are close to the patient. They can then respond to 
provide CPR and use a nearby AED before the arrival of EMS providers on scene. PulsePoint does not routinely collate outcome on patients with OHCA for whom citizens are activated. As of 25 May 2016, the PulsePoint program has been adopted by more than 1600 EMS agencies that are primarily based in the USA. (Price R. Personal communication, 25 May 2016). PulsePoint is supported by a subscription-based funding model.

Lay use of AEDs before the arrival of EMS providers on scene increases survival after OHCA. Large numbers of AEDs have been placed in public locations to improve the community response to cardiac arrest. These devices may be moved or may not be ready for use when needed. Our dynamic AED registry surveys the location of AEDs dynamically through time and space so as to improve the community response to and outcome of OHCA.

\section{Author affiliations}

${ }^{1}$ Department of Medicine, University of Washington-Harborview Center for Prehospital Emergency Care, University of Washington, Seattle, Washington, USA

${ }^{2}$ Salt Lake City Fire Department, University of Utah School of Medicine, Salt Lake City, Utah, USA

${ }^{3}$ Department of Surgery, University of Utah School of Medicine, Salt Lake City, Utah, USA

${ }^{4}$ Department of Emergency Medicine, Oregon Health and Sciences University, Portland, Oregon, USA

${ }^{5}$ Department of Emergency Medicine, University of Pittsburgh, Pittsburgh, Pennsylvania, USA

${ }^{6}$ Tucson Fire Department, University of Arizona, Tucson, Arizona, USA

${ }^{7}$ Department of Emergency Medicine, University of Arizona, Tucson, Arizona, USA

${ }^{8}$ Department of Emergency Medicine, University of Pennsylvania,

Pennsylvania, USA

${ }^{9}$ Penn Medicine Social Media and Health Innovation Laboratory, University of Pennsylvania, Philadelphia, Pennsylvania, USA

Twitter Follow Graham Nichol @grahamnichol

Collaborators Ben Eloff, PhD.

Contributors GN and RM contributed to the study conception and design. MD, SY, DS, TV and RM contributed to acquisition of data. GN, JBE, MD, SY, DS, TV and RM participated in drafting the article, revising it for critically important intellectual content and gave final approval of the submitted version.

Funding This work is supported by 1U01FD004587-04 from the Food and Drug Administration, Silver Spring, Maryland and ZOLL Medical Corporation, Chelmsford, MA

Competing interests The Dynamic AED Registry is funded by the Food and Drug Administration, Silver Spring, Maryland and ZOLL Medical Corporation, Chelmsford, Massachusetts.

Provenance and peer review Not commissioned; externally peer reviewed.

Open Access This is an Open Access article distributed in accordance with the Creative Commons Attribution Non Commercial (CC BY-NC 4.0) license, which permits others to distribute, remix, adapt, build upon this work noncommercially, and license their derivative works on different terms, provided the original work is properly cited and the use is non-commercial. See: http:// creativecommons.org/licenses/by-nc/4.0/

\section{REFERENCES}

1. Jacobs I, Nadkarni V, Bahr J, et al. Cardiac Arrest and Cardiopulmonary Resuscitation Outcome Reports: Update and Simplification of the Utstein Templates for Resuscitation Registries.
A Statement for Healthcare Professionals from a Task Force of the International Liaison Committee on Resuscitation (American Heart Association, European Resuscitation Council, Australian Resuscitation Council, New Zealand Resuscitation Council, Heart and Stroke Foundation of Canada, Interamerican Heart Foundation, Resuscitation Council of Southern Africa). Resuscitation 2004;63:233-49.

2. Holmberg M, Holmberg S, Herlitz J. An alternative estimate of the disappearance rate of ventricular fibrillation in our-of-hospital cardiac arrest in Sweden. Resuscitation 2001;49:219-20.

3. Valenzuela TD, Roe DJ, Cretin S, et al. Estimating effectiveness of cardiac arrest interventions: a logistic regression survival model. Circulation 1997;96:3308-13.

4. Hallstrom AP, Ornato JP, Weisfeldt M, et al. Public-access defibrillation and survival after out-of-hospital cardiac arrest. N Engl $J$ Med 2004;351:637-46.

5. Nichol G, Elrod JA, Becker LB. Treatment for out-of-hospital cardiac arrest: is the glass half empty or half full? Circulation 2014; 130:1844-6.

6. https://www.Federalregister.Gov/Documents/2015/01/29/2015-01619/ Effective-Date-of-Requirement-for-Premarket-Approval-forAutomated-External-Defibrillator-Systems (27 Sep 2016).

7. http://www.Fda.gov/Downloads/Advisorycommitees/Committe esmeetingmaterials/Medi caldevices/Medicaldevicesadvisory committee/Circulatorysystemdevicespanel/Ucm241780.pdf

8. Mozaffarian D, Benjamin EJ, Go AS, et al. Heart disease and stroke statistics-2016 update: a report from the American Heart Association. Circulation 2016;133:e38-e360.

9. Merchant RM, Asch DA, Hershey JC, et al. A crowdsourcing innovation challenge to locate and map automated external defibrillators. Circ Cardiovasc Qual Outcomes 2013;6:229-36.

10. Tang JC, Cebrian M, Giacobe NA, et al. Reflecting on the Darpa Red Balloon Challenge. Commun ACM 2011;54:78-85.

11. http://www.fda.gov/MedicalDevices/DeviceRegulationandGuidance/ UniqueDeviceldentification/default.htm (25 May 2016).

12. Morrison LJ, Nichol G, Rea TD, et al. Rationale, development and implementation of the Resuscitation Outcomes Consortium Epistry-Cardiac Arrest. Resuscitation 2008;78:161-9.

13. Nichol G, Thomas E, Callaway CW, et al. Regional variation in out-of-hospital cardiac arrest incidence and outcome. JAMA 2008;300:1423-31.

14. Daya MR, Schmicker RH, Zive DM, et al. Out-of-hospital cardiac arrest survival improving over time: results from the Resuscitation Outcomes Consortium (ROC). Resuscitation 2015;91:108-15.

15. Hostler D, Everson-Stewart S, Rea TD, et al. Effect of real-time feedback during cardiopulmonary resuscitation outside hospital: prospective, cluster-randomised trial. BMJ 2011;342:d512.

16. Stiell IG, Nichol G, Leroux BG, et al. Early versus later rhythm analysis in patients with out-of-hospital cardiac arrest. N Engl J Med 2011;365:787-97.

17. Nichol G, Leroux B, Wang $\mathrm{H}$, et al. Trial of continuous or interrupted chest compressions during CPR. N Engl J Med 2015;373:2203-14.

18. Ferreira-Gonzalez I, Marsal JR, Mitjavila F, et al. Patient registries of acute coronary syndrome: assessing or biasing the clinical real world data? Circ Cardiovasc Qual Outcomes 2009;2:540-7.

19. Krumholz HM. Registries and selection bias: the need for accountability. Circ Cardiovasc Qual Outcomes 2009;2:517-8.

20. Rea TD, Cook AJ, Stiell IG, et al. Predicting survival after out-of-hospital cardiac arrest: role of the Utstein data elements. Ann Emerg Med 2010;55:249-57.

21. http://www.FDA.gov/Medicaldevices/Deviceregulationandguidance/ Overview/Classifyyourdevice/Ucm051512.htm (27 Sep 2016).

22. Platt R, Wilson M, Chan KA, et al. The New Sentinel Network-the evidence of medical-product safety. N Engl J Med 2009;361:645-7.

23. http://www.Grassley.Senate.Gov/News/News-Releases/ Qa-Medical-Device-Safety (25 May 2016).

24. Institute of Medicine (US). Committee on the Public Health Effectiveness of the FDA 510(k) Clearance Process. Medical devices and the public's health: the FDA $510(K)$ clearance process at 35 years. Washington (DC): National Academies Press, 2011:298.

25. IOM (Institute of Medicine). Strategies to improve cardiac arrest survival: $A$ time to act. Washington, DC: The National Academies Press, 2015:458.

26. Kerber RE, Becker LB, Bourland JD, et al. Automatic external defibrillators for public access defibrillation: recommendations for specifying and reporting arrhythmia analysis algorithm performance, incorporating new waveforms, and enhancing safety. A Statement for Health Professionals from the American Heart Association Task Force on Automatic External Defibrillation, Subcommittee on AED Safety and Efficacy. Circulation 1997;95:1677-82. 
27. Nishiyama C, Brown SP, May S, et al. Apples to apples or apples to oranges? International variation in reporting of process and outcome of care for out-of-hospital cardiac arrest. Resuscitation 2014;85:1599-609.

28. Roger VL, Go AS, Lloyd-Jones DM, et al. Heart disease and stroke statistics-2012 update: a report from the American Heart Association. Circulation 2012;125:e2-e220.

29. Aufderheide TP, Kudenchuk PJ, Hedges JR, et al. Resuscitation Outcomes Consortium (ROC) PRIMED cardiac arrest trial methods part 1: rationale and methodology for the impedance threshold device (ITD) protocol. Resuscitation 2008;78:179-85.

30. Stiell IG, Callaway C, Davis D, et al. Resuscitation Outcomes Consortium (ROC) primed cardiac arrest trial methods. Part 2 rationale and methodology for "analyze later vs. analyze early" protocol. Resuscitation 2008;78:186-95.

31. Kudenchuk PJ, Brown SP, Daya M, et al. Amiodarone, lidocaine, or placebo in out-of-hospital cardiac arrest. N Engl J Med 2016;374:1711-22.

32. Cheskes S, Schmicker RH, Verbeek PR, et al. The impact of peri-shock pause on survival from out-of-hospital shockable cardiac arrest during the Resuscitation Outcomes Consortium Primed Trial. Resuscitation 2014;85:336-42.

33. Cheskes S, Schmicker RH, Christenson J, et al. Perishock pause: an independent predictor of survival from out-of-hospital shockable cardiac arrest. Circulation 2011;124:58-66.

34. Stiell IG, Brown SP, Christenson J, et al. What is the role of chest compression depth during out-of-hospital cardiac arrest resuscitation? Crit Care Med 2012;40:1192-8.

35. Stiell IG, Brown SP, Nichol G, et al. What is the optimal chest compression depth during out-of-hospital cardiac arrest resuscitation of adult patients? Circulation 2014;130:1962-70.
36. Idris AH, Guffey D, Aufderheide TP, et al. Relationship between chest compression rates and outcomes from cardiac arrest. Circulation 2012;125:3004-12.

37. Idris AH, Guffey D, Pepe PE, et al. Chest compression rates and survival following out-of-hospital cardiac arrest. Crit Care Med 2015;43:840-8.

38. Christenson J, Andrusiek D, Everson-Stewart S, et al. Chest compression fraction determines survival in patients with out-of-hospital ventricular fibrillation. Circulation 2009;120:1241-7.

39. Vaillancourt C, Everson-Stewart S, Christenson J, et al. The impact of increased chest compression fraction on return of spontaneous circulation for out-of-hospital cardiac arrest patients not in ventricular fibrillation. Resuscitation 2011;82:1501-7.

40. Glover BM, Brown SP, Morrison L, et al. Wide variability in drug use in out-of-hospital cardiac arrest: a report from the Resuscitation Outcomes Consortium. Resuscitation 2012;83:1324-30.

41. McNally B, Stokes A, Crouch A, et al. Cares: cardiac arrest registry to enhance survival. Ann Emerg Med 2009;54:674-83 e2.

42. Drory $\mathrm{Y}$, Turetz $\mathrm{Y}$, Hiss $\mathrm{Y}$, et al. Sudden unexpected death in persons less than 40 years of age. Am J Cardiol 1991;68:1388-92.

43. Kuisma M, Alaspaa A. Out-of-hospital cardiac arrests of non-cardiac origin. Epidemiology and outcome. Eur Heart J 1997;18:1122-8.

44. Vellano K, Crouch A, Rajdev M, et al. Cardiac arrest registry to enhance survival (cares) report on the public health burden of out-of-hospital cardiac arrest. Prepared for Institute of Medicine. 15 May 2015 [internet], 2016.

45. Dawson DE. National Emergency Medical Services Information System (NEMSIS). Prehosp Emerg Care 2006;10:314-16.

46. http://Nemsis.Org/Theproject/Whatisnemsis/Goalsandobjectives.html (17 June 2016) 